\title{
AN EFFICIENT COMPUTATIONAL METHOD FOR STATISTICAL MOMENTS OF BURGER'S EQUATION WITH RANDOM INITIAL CONDITIONS
}

\author{
HONGJOONG KIM
}

Received 15 November 2005; Revised 4 July 2006; Accepted 12 September 2006

The paper is concerned with efficient computation of numerical solutions to Burger's equation with random initial conditions. When the Lax-Wendroff scheme (LW) is expanded using the Wiener chaos expansion (WCE), random and deterministic effects can be separated and we obtain a system of deterministic equations with respect to HermiteFourier coefficients. One important property of the system is that all the statistical moments of the solution to the Burger's equation can be computed using the solution of the system only. Thus LW with WCE presents an alternative to computing moments by the Monte Carlo method (MC). It has been numerically demonstrated that LW with WCE approach is equally accurate but substantially faster than $\mathrm{MC}$ at least for certain classes of initial conditions.

Copyright (c) 2006 Hongjoong Kim. This is an open access article distributed under the Creative Commons Attribution License, which permits unrestricted use, distribution, and reproduction in any medium, provided the original work is properly cited.

\section{Introduction}

Uncertainty is observed in many and various phenomena in engineering, physics, biology, or finance. For example, small-scale effects in multiphase flow [13, 14] or Navier-Stokes equations $[18,26]$ may not be completely known, but subject to some random environmental effects. Or observational errors from inaccurate measurements may not be removed. Long waves of relatively shallow depth driven by white noise can be described approximately by Korteweg-de Vries equation with stochastic perturbations [8, 30]. Option pricing in financial markets may also introduce randomness $[4,10]$. These uncertainties are represented mathematically by (functions of) random variables or stochastic processes. Then, the governing equation is given in the form of a stochastic partial differential equation (SPDE). Since the solution of this type of equation is random, one particular solution corresponding to a specific realization of a random variable is not of

Hindawi Publishing Corporation

Mathematical Problems in Engineering

Volume 2006, Article ID 17406, Pages 1-21

DOI 10.1155/MPE/2006/17406 
concern. Instead, it is important to know the statistical properties of the solution such as its mean, variance, or higher-order moments.

Wiener chaos expansion (WCE) is a Fourier expansion with respect to the randomness $[3,15,19,23,24]$. In this paper, it is expanded with respect to the Gaussian random variable. The WCE of a solution to the Burger's equation, written by $v(x, t, \xi)$, is given by

$$
v(x, t, \xi)=\sum_{n=0}^{\infty} \frac{1}{\sqrt{n !}} v^{n}(x, t) \xi_{n}(\xi),
$$

where $\xi_{n}(x)$ is the $n$ th-order Wick polynomial. As explained in Section 2, Wick polynomials are complete orthogonal basis in the Wiener space and the series in the right-hand side of (1.1) converges in $L_{2}(R, \mu)$, where the Gaussian measure $\mu(d x)=(1 / \sqrt{2 \pi}) \exp \left\{-x^{2} /\right.$ $2\} d x$. This polynomial chaos method has been used by many authors $[5-7,12,17,33]$. As every Fourier expansion will do, WCE separates variables. More specifically, it separates nonrandom variables $(x, t)$ from the random one $\xi$. Then nonrandom Hermite-Fourier coefficients satisfy an infinite system of deterministic equations with nonlinearity similar to the one in Burger's equation. This system for the Hermite-Fourier coefficients is usually referred to as propagator, because it governs the propagation of randomness by the deterministic dynamics of the equation. One important property of the propagator is that all statistical moments of the solution to the Burger's equation can be computed by simple formulae that involve only the solution of the propagator. In addition, since the propagator is deterministic, it needs to be solved only once. Therefore, WCE presents an alternative to computing moments by the Monte Carlo (MC) method. There are two main issues addressed by the paper. First, a version of Lax-Wendroff scheme for the propagator of the Burger's equation has been developed. Second, the effectiveness of the WCE approach to computing statistical moments has been tested numerically and compared to the MC. A strong evidence has been demonstrated that suggested scheme is equally accurate but substantially faster than $\mathrm{MC}$ at least for certain initial conditions.

The Navier-Stokes (NS) equation is one of fundamental problems in fluid mechanics [20]. When the turbulence, for example, is modeled using a random variable, the NS is expressed as a stochastic equation $[15,18]$. Bensoussan and Temam initiated analytical study of an NS equation driven by a random force in [1]. Then, $[2,9,25,26]$ and many other researchers extended analytical studies of a stochastic Navier-Stokes (SNS) equation. But, there has not been many researches on numerical methods to solve an SNS. The MC is a classical way to solve an SPDE numerically [27, 31]. The MC may require a huge amount of computational time in order to obtain reliable estimation of statistical properties. Importance sampling or variance reduction method may be applied to reduce the workload of the MC $[11,22]$. Selection of random number generators is another weakness of the MC. As a preliminary step to an SNS, an inviscid Burger's equation with a random initial condition is considered in the present study. As seen below, the method can be generalized to an SNS. See also $[16,26]$.

The paper is organized as follows. Section 2 introduces Hermite polynomials and their properties. A version of Lax-Wendroff scheme for the propagator to the Burger's equation with random initial condition is derived in Section 3 using the WCE. Numerical results of the WCE approach to computing statistical moments are shown in Section 4. 


\section{Theoretical background}

The inner product of two real-valued functions $F(x)$ and $G(x)$ is defined by $\langle F, G\rangle_{w} \equiv$ $\int_{-\infty}^{\infty} F(x) G(x) w(x) \mathrm{d} x$, where the Gaussian probability density function, $w(x)=(1 /$ $\sqrt{2 \pi}) e^{-x^{2} / 2}$, is given as a weight function. Let $\|G(x)\|_{w} \equiv \sqrt{\langle G, G\rangle_{w}}$ be the induced norm from the inner product. The Gram-Schmidt process with respect to the norm applied to the sequence of functions defined by

$$
1, x, x^{2}, \ldots
$$

gives the orthonormal sequence $\left\{\xi_{n}\right\}$, where

$$
\begin{gathered}
\xi_{n}(x)=\frac{1}{\sqrt{n !}} H_{n}(x), \\
H_{0}(x)=1, \quad H_{n}(x)=(-1)^{n} e^{x^{2} / 2}\left(\frac{\mathrm{d}^{n}}{\mathrm{~d} x^{n}} e^{-x^{2} / 2}\right) .
\end{gathered}
$$

For example, $H_{1}(x)=x, H_{2}(x)=x^{2}-1, H_{3}(x)=x^{3}-3 x . H_{n}$ and $\xi_{n}$ are called the Hermite and Wick polynomials of order $n$, respectively. From the definition, the following relation can be easily derived:

$$
H_{n}^{\prime}=n H_{n-1}=x H_{n}-H_{n+1}
$$

Theorem 2.1 (see $[3,15]) .\left\{\xi_{n}(x)\right\}$ is a complete orthonormal set in $L^{2}(-\infty, \infty)$ with respect to the weight function $w(x)=(1 / \sqrt{2 \pi}) e^{-x^{2} / 2}$.

Reference [29] shows that a Fourier expansion in (1.1) converges uniformly.

Theorem 2.2 (see [29]). Let $f(x)$ be defined for all finite values of $x$ and integrable in any finite interval. Let $F(x)=F(0)+\int_{0}^{x} f(t) \mathrm{d} t,-\infty<x<\infty$. If $F(x)=O\left(e^{k x^{2} / 2}\right), 0<k<1$, and $\|-x F(x)+f(x)\|_{w}^{2}$ is convergent, then $\sum_{n=0}^{N}\left\langle F, \xi_{n}\right\rangle_{w} \xi_{n}(x) w(x)$ converges uniformly to $F(x) w(x)$ in $(-\infty, \infty)$.

Since $\left\{H_{n}\right\}$ is a basis, the product $H_{\alpha}(x) H_{\beta}(x)$ can be represented with respect to $\left\{H_{n}\right\}$, and the following lemma can be proved by induction.

Lemma 2.3. For nonnegative integers $\alpha$ and $\beta$,

$$
H_{\alpha}(x) H_{\beta}(x)=\sum_{\gamma=0}^{\alpha \wedge \beta} \gamma !\left(\begin{array}{l}
\alpha \\
\gamma
\end{array}\right)\left(\begin{array}{l}
\beta \\
\gamma
\end{array}\right) H_{\alpha+\beta-2 \gamma}(x)
$$

From Lemma 2.3 , for constant $u_{\alpha}$ and $v_{\alpha}(\alpha, \beta=0,1,2, \ldots)$,

$$
\left(\sum_{\alpha=0}^{\infty} \frac{u_{\alpha}}{\alpha !} H_{\alpha}(x)\right)\left(\sum_{\beta=0}^{\infty} \frac{v_{\beta}}{\beta !} H_{\beta}(x)\right)=\sum_{\alpha=0}^{\infty} \frac{u_{\alpha}}{\alpha !} \sum_{\beta=0}^{\infty} \frac{v_{\beta}}{\beta !} \sum_{\gamma=0}^{\alpha \wedge \beta} \gamma !\left(\begin{array}{l}
\alpha \\
\gamma
\end{array}\right)\left(\begin{array}{l}
\beta \\
\gamma
\end{array}\right) H_{\alpha+\beta-2 \gamma}(x) .
$$


4 Efficient computation of stochastic Burger's equation

By letting $(\alpha, \beta, \gamma)=(m+k, m+n-k, m)$,

$$
\begin{aligned}
\left(\sum_{\alpha=0}^{\infty} \frac{u_{\alpha}}{\alpha !} H_{\alpha}(x)\right)\left(\sum_{\beta=0}^{\infty} \frac{v_{\beta}}{\beta !} H_{\beta}(x)\right) \\
\quad=\sum_{n=0}^{\infty} \sum_{m=0}^{\infty} \sum_{k=0}^{n} \frac{u_{m+k}}{(m+k) !} \frac{v_{m+n-k}}{(m+n-k) !} m !\left(\begin{array}{c}
m+k \\
m
\end{array}\right)\left(\begin{array}{c}
m+n-k \\
m
\end{array}\right) H_{n}(x) \\
=\sum_{n=0}^{\infty} \frac{1}{n !} \sum_{m=0}^{\infty} \frac{1}{m !} \sum_{k=0}^{n}\left(\begin{array}{l}
n \\
k
\end{array}\right) u_{m+k} v_{m+n-k} H_{n}(x) .
\end{aligned}
$$

Thus the product of two series in Hermite polynomials can be simplified as follows.

Lemma 2.4 .

$$
\left(\sum_{\alpha=0}^{\infty} \frac{u_{\alpha}}{\alpha !} H_{\alpha}(x)\right)\left(\sum_{\beta=0}^{\infty} \frac{v_{\beta}}{\beta !} H_{\beta}(x)\right)=\sum_{n=0}^{\infty} \frac{1}{n !} \sum_{m=0}^{\infty} \frac{1}{m !} \sum_{k=0}^{n}\left(\begin{array}{l}
n \\
k
\end{array}\right) u_{m+k} v_{m+n-k} H_{n}(x)
$$

where $u_{\alpha}$ and $v_{\alpha}$ are constants for all $\alpha$.

\section{Derivation of numerical algorithm}

Let us consider the following conservation law for $v(x, t, \xi)$ with a random initial condition:

$$
v_{t}+f(v)_{x}=0, \quad v(x, 0, \xi)=g(x, \xi)
$$

where $f(v)=(1 / 2) v^{2}$ and $g(x, \xi)$ is a function of $x$ and $\xi$. $\xi$ is a Gaussian random variable $N(0,1)$ with zero mean and unit variance. The WCE is a Fourier expansion with respect to the randomness. In this paper, it represents the solution $v=v(x, t, \xi)$ of $(3.1)$ as a series in $\xi$ with respect to the Wick polynomials

$$
v(x, t, \xi)=\sum_{n=0}^{\infty} \frac{1}{\sqrt{n !}} v^{n}(x, t) \xi_{n}(\xi)
$$

where $v^{n}(x, t)=\sqrt{n !}\left\langle v(x, t, \xi), \xi_{n}(\xi)\right\rangle_{w} . v^{n}$ is called the Hermite-Fourier coefficient of order $n$ of $v . H_{n}(\xi)$ and $\xi_{n}(\xi)$ will be hereafter denoted by $H_{n}$ and $\xi_{n}$, respectively, for notational simplicity.

When $v(x, t, \xi)$ in (3.1) is expanded using a series (3.2), we obtain the following from Lemmas 2.3 and 2.4:

$$
v v_{x}=\sum_{n=0}^{\infty} \frac{1}{\sqrt{n !}} \sum_{m=0}^{\infty} \frac{1}{m !} \sum_{k=0}^{n}\left(\begin{array}{l}
n \\
k
\end{array}\right) v^{m+k} v_{x}^{m+n-k} \xi_{n}
$$


Then, from the orthogonality in Theorem 2.1, for every $n<\infty$, the Hermite-Fourier coefficient $v^{n}$ is a solution of the following system:

$$
\begin{gathered}
\frac{\partial}{\partial t} v^{n}(x, t)=-\sum_{m=0}^{\infty} \frac{1}{m !} \sum_{0 \leq k \leq n<\infty}\left(\begin{array}{l}
n \\
k
\end{array}\right) v^{m+k}(x, t) \frac{\partial}{\partial x} v^{m+n-k}(x, t) \\
v^{n}(x, 0)=\sqrt{n !} E\left[g(x, \xi) \xi_{n}\right]
\end{gathered}
$$

where $t \in(0, T]$ and $x \in R^{1}$. System (3.4) is referred to as propagator system because it governs the propagation of randomness. It separates nonrandom variables $(x, t)$ from the random one $\xi$. Since this propagator system (3.4) is deterministic, the system needs to be solved only once. Once the propagator is solved, statistical moments can be computed as follows. First, note that $E\left[\xi_{0}\right]=1$ and $E\left[\xi_{n}\right]=0$ for $n>0$, where $E$ is the expectation symbol. Thus, the first moment is obtained by

$$
E[v(x, t, \xi)]=\sum_{n=0}^{\infty} \frac{1}{\sqrt{n !}} v^{n}(x, t) E\left[\xi_{n}\right]=v^{0}(x, t),
$$

where the expectation and the limit can be interchanged because of the uniform convergence in Theorem 2.2. By Theorem 2.1 and Parseval's identity, the expression for the second moment can be derived as follows:

$$
E\left[v^{2}(x, t, \xi)\right]=\sum_{n=0}^{\infty} \frac{1}{n !}\left|v^{n}(x, t)\right|^{2}
$$

In a similar way, higher moments can be obtained as functions of Hermite-Fourier coefficients.

The Lax-Wendroff scheme (LW) for the deterministic conservation laws,

$$
v_{t}+f(v)_{x}=0
$$

computes $v_{j, i} \equiv v\left(x_{j}, t_{i}\right)$ by

$$
\begin{aligned}
v_{j, i+1}= & v_{j, i}-\frac{1}{2}\left(\frac{\Delta t}{\Delta x}\right)\left(\Delta_{+x} F_{j, i}+\Delta_{-x} F_{j, i}\right) \\
& +\frac{1}{2}\left(\frac{\Delta t}{\Delta x}\right)^{2}\left(A_{j+1 / 2, i} \Delta_{+x} F_{j, i}-A_{j-1 / 2, i} \Delta_{-x} F_{j, i}\right),
\end{aligned}
$$

where $F_{j, i} \equiv f\left(v_{j, i}\right)$ and $A_{j, i} \equiv(\partial f / \partial v)\left(v_{j, i}\right)[28] . \Delta_{+x} F_{j, i}$ and $\Delta_{-x} F_{j, i}$ are defined by $\left(F_{j+1, i}-\right.$ $\left.F_{j, i}\right)$ and $\left(F_{j, i}-F_{j-1, i}\right)$, respectively. $A_{j \pm 1 / 2, i}$ denotes $(1 / 2)\left(A_{j, i}+A_{j \pm 1, i}\right)$. When $v_{j, i}$ in (3.8) is expanded using a series in (3.2), the propagator system for each Hermite-Fourier coefficient $v_{j, i}^{n} \equiv v^{n}\left(x_{j}, t_{i}\right)$ can be solved by a following modified version of the Lax-Wendroff 
6 Efficient computation of stochastic Burger's equation

scheme (denoted by LW with WCE hereafter):

$$
\begin{aligned}
v_{j, i+1}^{n}= & v_{j, i}^{n}-\frac{1}{4}\left(\frac{\Delta t}{\Delta x}\right)\left(w_{j+1, i}^{n}-w_{j-1, i}^{n}\right) \\
& +\frac{1}{8}\left(\frac{\Delta t}{\Delta x}\right)^{2} \sum_{\alpha=0}^{\infty}\left\{\left(z_{j, i}^{n, \alpha}+z_{j+1, i}^{n, \alpha}\right)\left(w_{j+1, i}^{\alpha}-w_{j, i}^{\alpha}\right)-\left(z_{j, i}^{n, \alpha}+z_{j-1, i}^{n, \alpha}\right)\left(w_{j, i}^{\alpha}-w_{j-1, i}^{\alpha}\right)\right\},
\end{aligned}
$$

where

$$
\begin{gathered}
w_{j, i}^{\alpha}=\sum_{m=0}^{\infty} \frac{1}{m !}\left(2 \sum_{0 \leq k<\alpha / 2}\left(\begin{array}{l}
\alpha \\
k
\end{array}\right) v_{j, i}^{m+k} v_{j, i}^{m+\alpha-k}+\chi_{\{\alpha / 2=[\alpha / 2]\}}\left(\begin{array}{c}
\alpha \\
\frac{\alpha}{2}
\end{array}\right)\left(v_{j, i}^{m+\alpha / 2}\right)^{2}\right), \\
z_{j, i}^{n, \alpha}=\sum_{q=\max \{0, n-\alpha\}}^{n} \frac{1}{(\alpha-n+q) !}\left(\begin{array}{l}
n \\
q
\end{array}\right) v_{j, i}^{\alpha-n+2 q}
\end{gathered}
$$

$\chi_{\{a=b\}}=1$ if $a=b$, and 0 otherwise, and $[x]$ is the smallest integer, which is not smaller than $x$. It is important to notice that (3.9) separates $\left\{z_{j, i}^{n, \alpha}\right\}$ and $\left\{w_{j, i}^{\alpha}\right\}$, which removes unnecessary loops in computation. In addition, $\left\{w_{j, i}^{\alpha}\right\}$ get rid of repetitive calculations. Due to numerical limitations, algorithms with only a finite number of $\left\{v^{n}\right\}$ will be implemented. The number of $\left\{v^{n}\right\}$ used in the computation is called the length of the WCE. Equation (3.9) can be derived as follows: using (3.3),

$$
\Delta_{ \pm x} F_{j, i}=\frac{1}{2} \sum_{n=0}^{\infty} \frac{1}{\sqrt{n !}} \sum_{m=0}^{\infty} \frac{1}{m !} \sum_{k=0}^{n}\left(\begin{array}{l}
n \\
k
\end{array}\right) \psi_{j \pm 1 / 2, i}^{m, n, k} \xi_{n},
$$

where

$$
\psi_{j+1 / 2, i}^{m, n, k}=v_{j+1, i}^{m+k} v_{j+1, i}^{m+n-k}-v_{j, i}^{m+k} v_{j, i}^{m+n-k} .
$$

Then,

$$
\Delta_{+x} F_{j, i}+\Delta_{-x} F_{j, i}=\frac{1}{2} \sum_{n=0}^{\infty} \frac{1}{\sqrt{n !}} \sum_{m=0}^{\infty} \frac{1}{m !} \sum_{k=0}^{n}\left(\begin{array}{l}
n \\
k
\end{array}\right) \phi_{j, i}^{m, n, k} \xi_{n},
$$

where

$$
\phi_{j, i}^{m, n, k}=v_{j+1, i}^{m+k} v_{j+1, i}^{m+n-k}-v_{j-1, i}^{m+k} v_{j-1, i}^{m+n-k} .
$$

Also,

$$
A_{j+1 / 2, i}=\frac{1}{2} \sum_{\alpha=0}^{\infty} \frac{1}{\sqrt{\alpha !}} \varphi_{j+1 / 2, i}^{\alpha} \xi_{\alpha}
$$

where

$$
\varphi_{j+1 / 2, i}^{\alpha}=v_{j, i}^{\alpha}+v_{j+1, i}^{\alpha}
$$


Then,

$$
\begin{aligned}
A_{j+1 / 2, i} \Delta_{+x} F_{j, i} \\
=\left(\frac{1}{2} \sum_{\alpha=0}^{\infty} \frac{1}{\sqrt{\alpha !}} \varphi_{j+1 / 2, i}^{\alpha} \xi_{\alpha}\right)\left(\frac{1}{2} \sum_{\beta=0}^{\infty} \frac{1}{\sqrt{\beta !}} \sum_{m=0}^{\infty} \frac{1}{m !} \sum_{k=0}^{\beta}\left(\begin{array}{l}
\beta \\
k
\end{array}\right) \psi_{j+1 / 2, i}^{m, \beta, k} \xi_{\beta}\right) \\
=\frac{1}{4} \sum_{\alpha=0}^{\infty} \frac{1}{\alpha !} \varphi_{j+1 / 2, i}^{\alpha} \sum_{\beta=0}^{\infty} \frac{1}{\beta !} \sum_{m=0}^{\infty} \frac{1}{m !} \sum_{k=0}^{\beta}\left(\begin{array}{l}
\beta \\
k
\end{array}\right) \psi_{j+1 / 2, i}^{m, \beta, k} \sum_{\gamma=0}^{\alpha \wedge \beta} \gamma !\left(\begin{array}{l}
\alpha \\
\gamma
\end{array}\right)\left(\begin{array}{l}
\beta \\
\gamma
\end{array}\right) H_{\alpha+\beta-2 \gamma} .
\end{aligned}
$$

When $(\alpha, \beta, \gamma)$ are replaced by $(p+q, p+n-q, p)$,

$$
\begin{aligned}
A_{j+1 / 2, i} & \Delta_{+x} F_{j, i} \\
= & \frac{1}{4} \sum_{n=0}^{\infty} \sum_{p=0}^{\infty} \sum_{q=0}^{n} \frac{1}{(p+q) !} \varphi_{j+1 / 2, i}^{p+q} \frac{1}{(p+n-q) !} \sum_{m=0}^{\infty} \frac{1}{m !} \sum_{k=0}^{p+n-q}\left(\begin{array}{c}
p+n-q \\
k
\end{array}\right) \psi_{j+1 / 2, i}^{m, p+n-q, k} \\
& \times p !\left(\begin{array}{c}
p+q \\
p
\end{array}\right)\left(\begin{array}{c}
p+n-q \\
p
\end{array}\right) H_{n} \\
= & \frac{1}{4} \sum_{n=0}^{\infty} \frac{1}{\sqrt{n !}} \sum_{p=0}^{\infty} \frac{1}{p !} \sum_{q=0}^{n}\left(\begin{array}{c}
n \\
q
\end{array}\right) \varphi_{j+1 / 2, i}^{p+q} \sum_{m=0}^{\infty} \frac{1}{m !} \sum_{k=0}^{p+n-q}\left(\begin{array}{c}
p+n-q \\
k
\end{array}\right) \psi_{j+1 / 2, i}^{m, p+n-q, k} \xi_{n} .
\end{aligned}
$$

When (3.8) is expanded using these series,

$$
\begin{aligned}
& \sum_{n=0}^{\infty} \frac{1}{\sqrt{n !}} v_{j, i+1}^{n} \xi_{n} \\
&=\sum_{n=0}^{\infty} \frac{1}{\sqrt{n !}} v_{j, i}^{n} \xi_{n} \\
& \quad-\frac{1}{4}\left(\frac{\Delta t}{\Delta x}\right) \sum_{n=0}^{\infty} \frac{1}{\sqrt{n !}} \sum_{m=0}^{\infty} \frac{1}{m !} \sum_{k=0}^{n}\left(\begin{array}{l}
n \\
k
\end{array}\right) \phi_{j, i}^{m, n, k} \xi_{n} \\
& \quad+\frac{1}{8}\left(\frac{\Delta t}{\Delta x}\right)^{2} \sum_{n=0}^{\infty} \frac{1}{\sqrt{n !}} \sum_{p=0}^{\infty} \frac{1}{p !} \sum_{q=0}^{n}\left(\begin{array}{l}
n \\
q
\end{array}\right) \varphi_{j+1 / 2, i}^{p+q} \sum_{m=0}^{\infty} \frac{1}{m !} \sum_{k=0}^{p+n-q}\left(\begin{array}{c}
p+n-q \\
k
\end{array}\right) \psi_{j+1 / 2, i}^{m, p+n-q, k} \xi_{n} \\
&-\frac{1}{8}\left(\frac{\Delta t}{\Delta x}\right)^{2} \sum_{n=0}^{\infty} \frac{1}{\sqrt{n !}} \sum_{p=0}^{\infty} \frac{1}{p !} \sum_{q=0}^{n}\left(\begin{array}{l}
n \\
q
\end{array}\right) \varphi_{j-1 / 2, i}^{p+q} \sum_{m=0}^{\infty} \frac{1}{m !} \sum_{k=0}^{p+n-q}\left(\begin{array}{c}
p+n-q \\
k
\end{array}\right) \psi_{j-1 / 2, i}^{m, p+n-q, k} \xi_{n} .
\end{aligned}
$$


8 Efficient computation of stochastic Burger's equation

By comparing coefficients of $\xi_{n}$, we obtain from orthogonality

$$
\begin{aligned}
v_{j, i+1}^{n}= & v_{j, i}^{n}-\frac{1}{4}\left(\frac{\Delta t}{\Delta x}\right) \sum_{m=0}^{\infty} \frac{1}{m !} \sum_{k=0}^{n}\left(\begin{array}{l}
n \\
k
\end{array}\right) \phi_{j, i}^{m, n, k} \\
& +\frac{1}{8}\left(\frac{\Delta t}{\Delta x}\right)^{2} \sum_{p=0}^{\infty} \frac{1}{p !} \sum_{q=0}^{n}\left(\begin{array}{l}
n \\
q
\end{array}\right) \varphi_{j+1 / 2, i}^{p+q} \sum_{m=0}^{\infty} \frac{1}{m !} \sum_{k=0}^{p+n-q}\left(\begin{array}{c}
p+n-q \\
k
\end{array}\right) \psi_{j+1 / 2, i}^{m, p+n-q, k} \\
& -\frac{1}{8}\left(\frac{\Delta t}{\Delta x}\right)^{2} \sum_{p=0}^{\infty} \frac{1}{p !} \sum_{q=0}^{n}\left(\begin{array}{l}
n \\
q
\end{array}\right) \varphi_{j-1 / 2, i}^{p+q} \sum_{m=0}^{\infty} \frac{1}{m !} \sum_{k=0}^{p+n-q}\left(\begin{array}{c}
p+n-q \\
k
\end{array}\right) \psi_{j-1 / 2, i}^{m, p+n-q, k} .
\end{aligned}
$$

Since (3.20) contains nested loops, it is not practical to use this equation directly to update $v_{j, i}^{n}$. Instead, let $\alpha=p+n-q$. Then,

$$
\begin{aligned}
& \sum_{p=0}^{\infty} \frac{1}{p !} \sum_{q=0}^{n}\left(\begin{array}{l}
n \\
q
\end{array}\right) \varphi_{j+1 / 2, i}^{p+q} \sum_{m=0}^{\infty} \frac{1}{m !} \sum_{k=0}^{p+n-q}\left(\begin{array}{c}
p+n-q \\
k
\end{array}\right) \psi_{j+1 / 2, i}^{m, p+n-q, k} \\
& \quad=\sum_{\alpha=0}^{\infty}\left(\sum_{q=\max \{0, n-\alpha\}}^{n} \frac{1}{(\alpha-n+q) !}\left(\begin{array}{l}
n \\
q
\end{array}\right) \varphi_{j+1 / 2, i}^{\alpha-n+2 q}\right)\left(\sum_{m=0}^{\infty} \frac{1}{m !} \sum_{k=0}^{\alpha}\left(\begin{array}{l}
\alpha \\
k
\end{array}\right) \psi_{j+1 / 2, i}^{m, \alpha, k}\right) .
\end{aligned}
$$

Note that the first term in the summation over $\alpha$ in the right-hand side of (3.21) is independent of $m$ and $k$ and that the second term is independent of $q$. This factorization removes unnecessary nested loops and saves computation. In addition,

$$
\begin{aligned}
& \sum_{m=0}^{\infty} \frac{1}{m !} \sum_{k=0}^{\alpha}\left(\begin{array}{l}
\alpha \\
k
\end{array}\right) \psi_{j+1 / 2, i}^{m, \alpha, k} \\
& =\sum_{m=0}^{\infty} \frac{1}{m !}\left\{2 \sum_{0 \leq k<\alpha / 2}\left(\begin{array}{l}
\alpha \\
k
\end{array}\right)\left(v_{j+1, i}^{m+k} v_{j+1, i}^{m+\alpha-k}-v_{j, i}^{m+k} v_{j, i}^{m+\alpha-k}\right)\right. \\
& \left.+\chi_{\{\alpha / 2=[\alpha / 2]\}}\left(\begin{array}{c}
\alpha \\
\frac{\alpha}{2}
\end{array}\right)\left(\left(v_{j+1, i}^{m+\alpha / 2}\right)^{2}-\left(v_{j, i}^{m+\alpha / 2}\right)^{2}\right)\right\} \\
& =w_{j+1, i}^{\alpha}-w_{j, i}^{\alpha} .
\end{aligned}
$$

Notice also that since $w_{j, i}^{\alpha}$ is independent of $n$, it can be computed even prior to $n$-loop. Now by definition of $z_{j, i}^{n, \alpha}$,

$$
\sum_{q=\max \{0, n-\alpha\}}^{n} \frac{1}{(\alpha-n+q) !}\left(\begin{array}{l}
n \\
q
\end{array}\right) \varphi_{j+1 / 2, i}^{\alpha-n+2 q}=z_{j, i}^{n, \alpha}+z_{j+1, i}^{n, \alpha} .
$$

Finally,

$$
\sum_{m=0}^{\infty} \frac{1}{m !} \sum_{k=0}^{n}\left(\begin{array}{l}
n \\
k
\end{array}\right) \phi_{j, i}^{m, n, k}=w_{j+1, i}^{\alpha}-w_{j-1, i}^{\alpha}
$$

completes the derivation of (3.9). 
Initial and boundary conditions can be changed for $v^{n}$ as well. An initial condition $v(x, 0, \xi)=g(x, \xi)$ can be written as

$$
g(x, \xi)=\sum_{n=0}^{\infty} \frac{1}{\sqrt{n !}} g^{n} \xi_{n},
$$

where $g^{n}=\sqrt{n !} E\left[g \xi_{n}\right]$ so that $g^{n}(x)$ becomes the initial condition for $v^{n}(x, t)$. Similarly, a boundary condition $v\left(x_{0}, t, \xi\right)=h(t, \xi)$ can be written as

$$
h(t, \xi)=\sum_{n=0}^{\infty} \frac{1}{\sqrt{n !}} h^{n} \xi_{n},
$$

where $h^{n}=\sqrt{n !} E\left[h \xi_{n}\right]$ and $h^{n}(t)$ is the boundary condition for $v^{n}(x, t)$ at $x=x_{0}$.

As [21] points out, for general systems of equations with arbitrary initial data, no numerical method has been proved to be stable or convergent in general, although convergence results have been obtained in some special cases. The stability of the LW with WCE in (3.9) is obtained by analyzing the stability of the scheme when the Jacobian of (3.1) is assumed to be a constant. Each Hermite-Fourier coefficient $v^{n}$, then, satisfies deterministic Lax-Wendroff in (3.8)

$$
\begin{aligned}
v^{n}\left(x_{j}, t_{i+1}\right)= & v^{n}\left(x_{j}, t_{i}\right)-\frac{1}{2}\left(\frac{a \Delta t}{\Delta x}\right)\left(v_{j+1, i}^{n}-v_{j-1, i}^{n}\right) \\
& +\frac{1}{2}\left(\frac{a \Delta t}{\Delta x}\right)^{2}\left(v_{j+1, i}^{n}-2 v_{j, i}^{n}+v_{j-1, i}^{n}\right),
\end{aligned}
$$

where $a$ is the constant Jacobian so that the stability condition is

$$
|\nu| \leq 1
$$

where $v=(\Delta t / \Delta x) \max v$ is the Courant number. More analytical study on stability and error analysis will follow in the future research. As can be seen in the derivation, (3.9) can be generalized to the propagator of a viscous Burger's equation or even an SNS.

\section{Numerical results}

The stability condition (3.28) for the LW with WCE is numerically examined in Section 4 first. Then, accuracy of the scheme is investigated with respect to the length of the WCE and the Courant number. The accuracy is also compared to that from the MC. Finally, the analysis on efficiency of the scheme is shown based on the computation time.

4.1. Stability. If (3.28) is satisfied, the LW with WCE does not show dispersion or diffusion when the initial condition is continuous. Examples for such a case will be shown in Section 4.2. In this section, let us consider a nonlinear inviscid Burger's equation with a 
following random discontinuous initial condition:

$$
\begin{aligned}
v_{t}(x, t, \xi)+\frac{\partial}{\partial x} f(v(x, t, \xi)) & =v_{t}(x, t, \xi)+v(x, t, \xi) v_{x}(x, t, \xi)=0 \\
v(-1, t, \xi)=1, & \\
v(x, 0, \xi) & = \begin{cases}1, & x<0, \\
\sin (x \xi), & x>0,\end{cases}
\end{aligned}
$$

where $x \in[-1,1]$ and $\xi \sim N(0,1)$ is a Gaussian random variable with mean zero and variance one. For each realization of $\xi$, shocks will be developed. The initial condition can be obtained from (3.25): for $x<0$,

$$
v^{n}(x, 0)= \begin{cases}1, & n=0 \\ 0, & n \neq 0\end{cases}
$$

and for $x>0$,

$$
v^{n}(x, 0)=E\left[\sin (x \xi) \xi_{n}(\xi)\right]= \begin{cases}\frac{(-1)^{m}}{\sqrt{(2 m+1) !}} x^{2 m+1} e^{-x^{2} / 2}, & n=2 m+1, \\ 0, & n=2 m .\end{cases}
$$

Figure 4.1 shows the first and second moments of solutions for (4.1) computed by the LW with WCE when the Courant number is 0.6 or 1.0. Both solutions are stable, but the one obtained with 0.6 is much less accurate due to the known deficiency of the LW near discontinuities. If moments of $v(x, t, \xi)$ are computed up to $t \leq T$, as the Courant number is reduced, more computations will be required to reach the time $T$ and the quality of the solution will be degraded, similarly to the deterministic LW [32].

It has been also found numerically that the stability condition becomes more conservative as the length of the WCE decreases, in the sense that the range of the Courant number for stability decreases as the length decreases. Table 4.1 shows maximum Courant numbers maintaining stability of the scheme for different lengths of the WCE.

4.2. Accuracy. When (4.1) is solved, accuracy analysis is limited due to dispersions. Thus, let us consider the Burger's equation with the following random initial condition:

$$
v(x, 0, \xi)=x \sin (2 \pi x)+\frac{x-1}{4} \sin (2 \xi x),
$$

where $x \in[0,1]$ and $\xi \sim N(0,1)$ is Gaussian. From (3.25),

$$
v^{n}(x, 0)= \begin{cases}x \sin (2 \pi x), & n=0, \\ \frac{(-1)^{m}}{\sqrt{(2 m+1) !}} \frac{(x-1)}{4}(2 x)^{2 m+1} e^{-2 x^{2}}, & n=2 m+1, \\ 0, & n=2 m \neq 0 .\end{cases}
$$




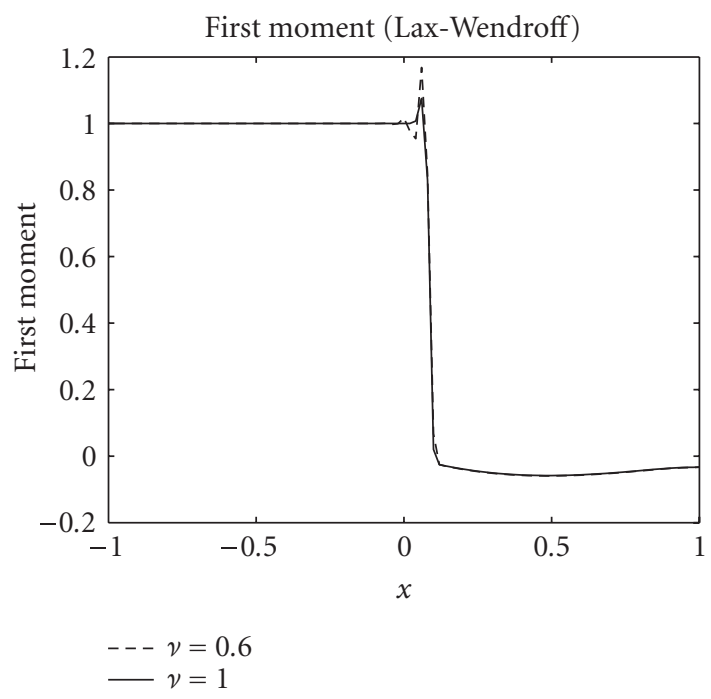

(a) First moment (Lax-Wendroff)

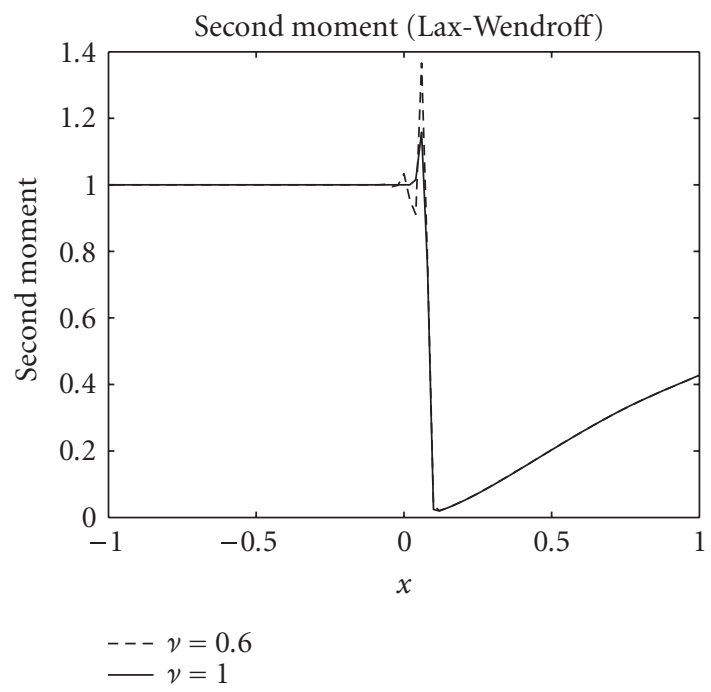

(b) Second moment (Lax-Wendroff)

Figure 4.1. First two moments from the LW with WCE when $v=0.6$ or 1.

Due to the nonlinearity, the statistical moments cannot be obtained analytically. When the $\mathrm{MC}$ is applied to the problem, statistical moments show fluctuations as the number of samples varies. The convergence has been obtained when the number of realizations exceeds $10^{6}$. Thus, the results from the MC with $10^{6}$ samples will be considered as exact 
Efficient computation of stochastic Burger's equation

Table 4.1. Maximum Courant numbers maintaining stability of the LW with WCE.

\begin{tabular}{lcccccccc}
\hline Length of WCE & 1 & 2 & 3 & 4 & 5 & 6 & 7 & 8 \\
\hline Courant number & 1.0 & 1.0 & 1.0 & 1.0 & 1.0 & 1.0 & 0.3 & 0.3 \\
\hline
\end{tabular}

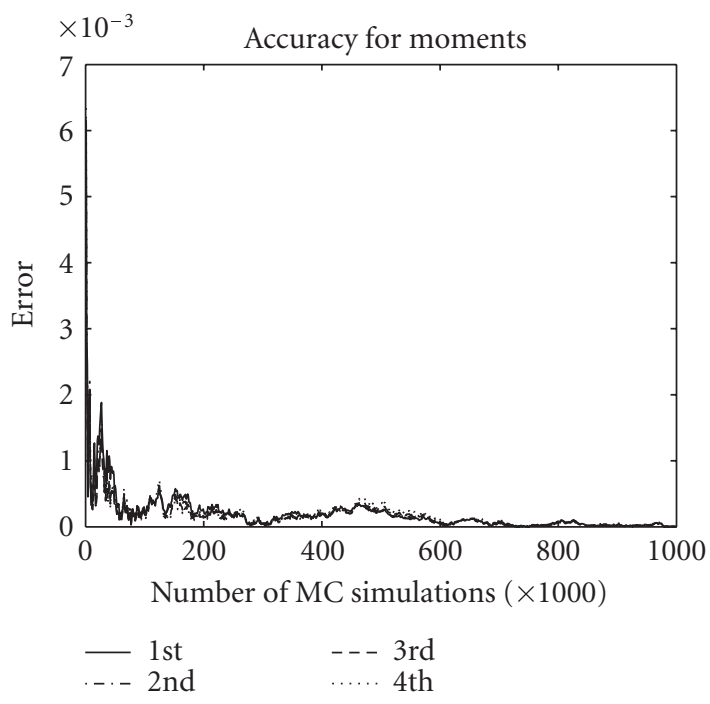

Figure 4.2. Errors of the MC in statistical moments as the number of simulations increases.

values in the present study. For the error comparison, a relative error is used, which is defined by

$$
\frac{\|u(x, t)-\tilde{u}(x, t)\|}{\|u(x, t)\|}
$$

where $u(x, t)$ is a statistical moment from the exact solution and $\tilde{u}(x, t)$ is an approximation.

Figure 4.2 shows relative errors of the MC in computing statistical moments of up to order 4 as the number of samples in the Monte Carlo simulation increases. Fluctuations of errors are observed in the figure even when the number of samples is large.

Figures 4.3 and 4.4 compare statistical moments of up to order 4 between the exact solution and the LW with WCE and show that very good accuracy can be obtained with sufficiently small lengths. Note also that dispersion or diffusion is not developed in Figures 4.3 and 4.4 .

Figures 4.5 and 4.6 show the differences between the statistical moments from the exact solution and those from the LW with WCE. Note that the scales in Figures 4.5 and 4.6 are much finer than those in Figures 4.3 and 4.4. 


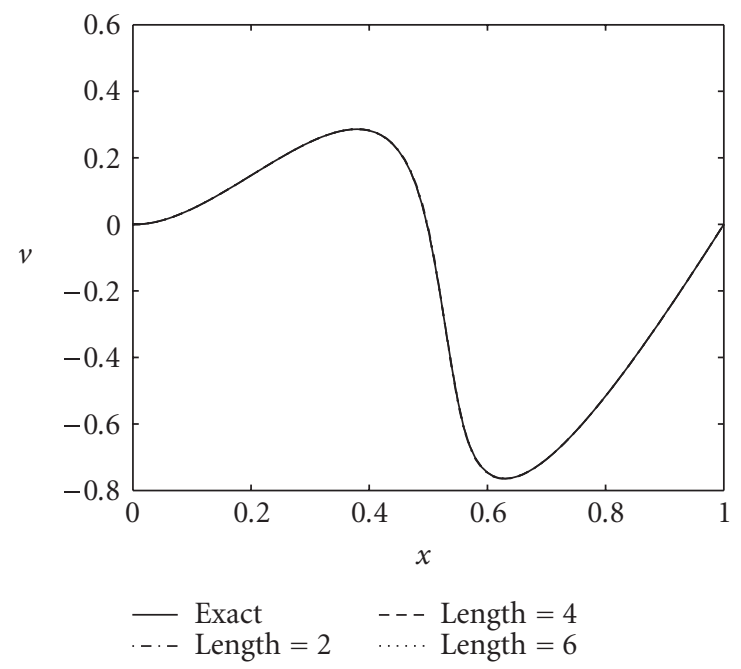

(a)

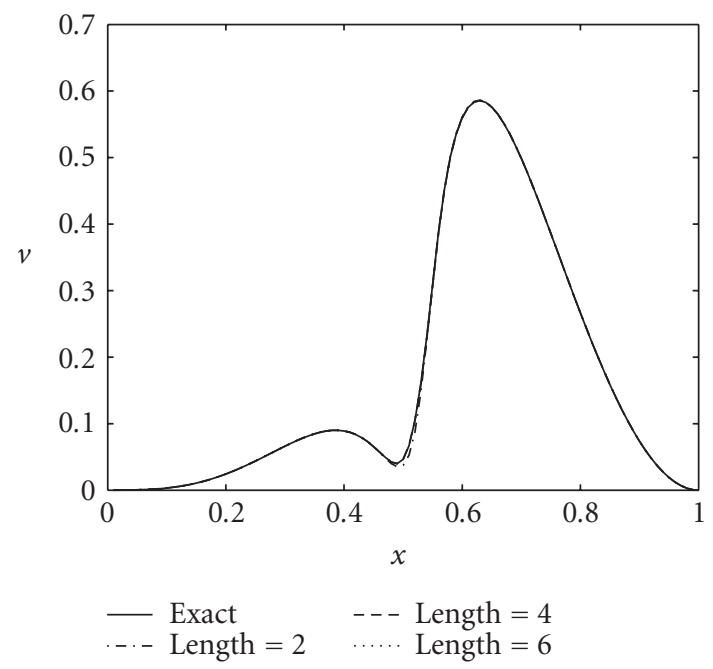

(b)

Figure 4.3. Comparison of the first (a) and second (b) moments of the exact solution with approximations from the LW with WCE for several lengths.

Figure 4.7 shows relative errors (4.6) from the LW with WCE in statistical moments with respect to lengths of the WCE. As the length increases, more Hermite-Fourier coefficients are introduced and better accuracy is obtained. The first moment depends on $v^{0}$ only and good accuracy is obtained even when the length is 3 . For the second, third, and 
14 Efficient computation of stochastic Burger's equation

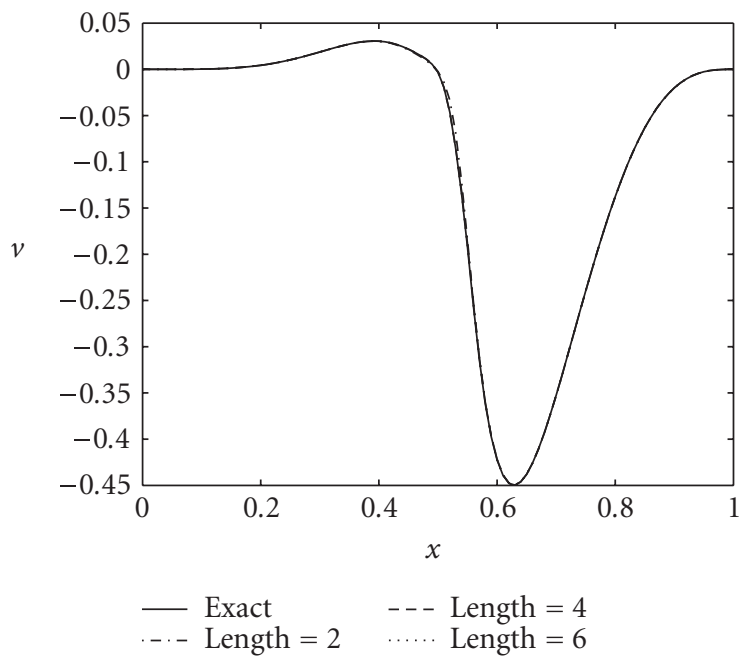

(a)

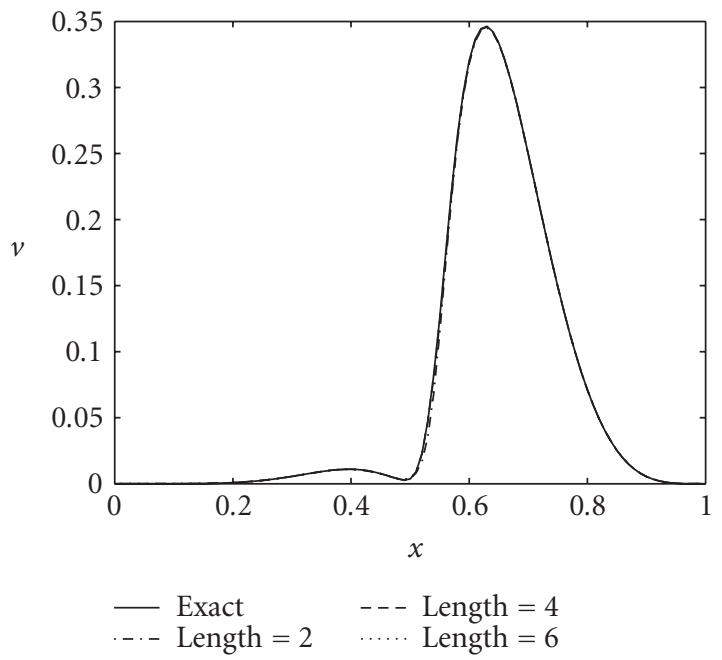

(b)

Figure 4.4. Comparison of the third (a) and fourth (b) moments of the exact solution with approximations from the LW with WCE for several lengths.

fourth moments, the convergence is obtained with length 6 , which is still small enough. Figure 4.8 shows relative errors from the LW with WCE for various Courant numbers.

4.3. Efficiency. In order to evaluate the efficiency, let us compare computation times of these numerical schemes for the same relative error. For example, when the length of the 


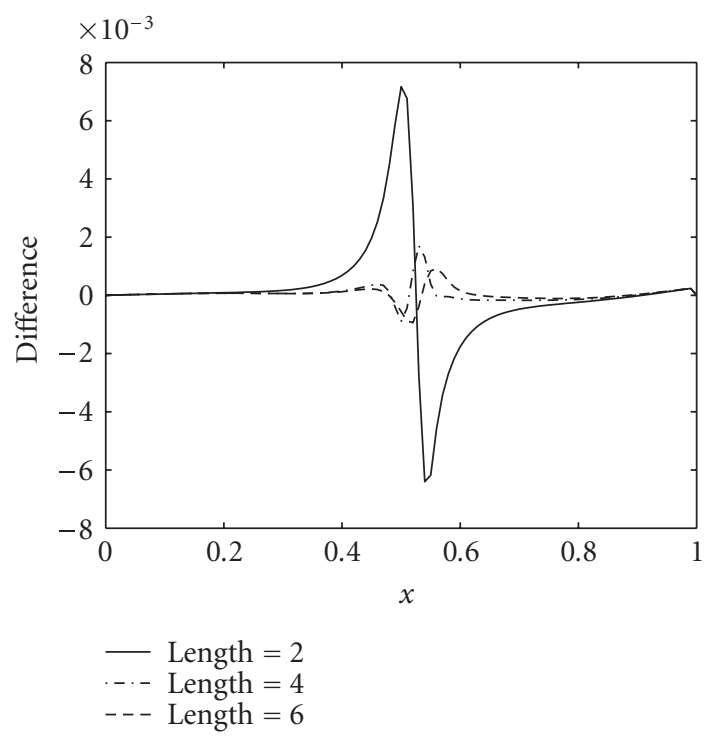

(a)

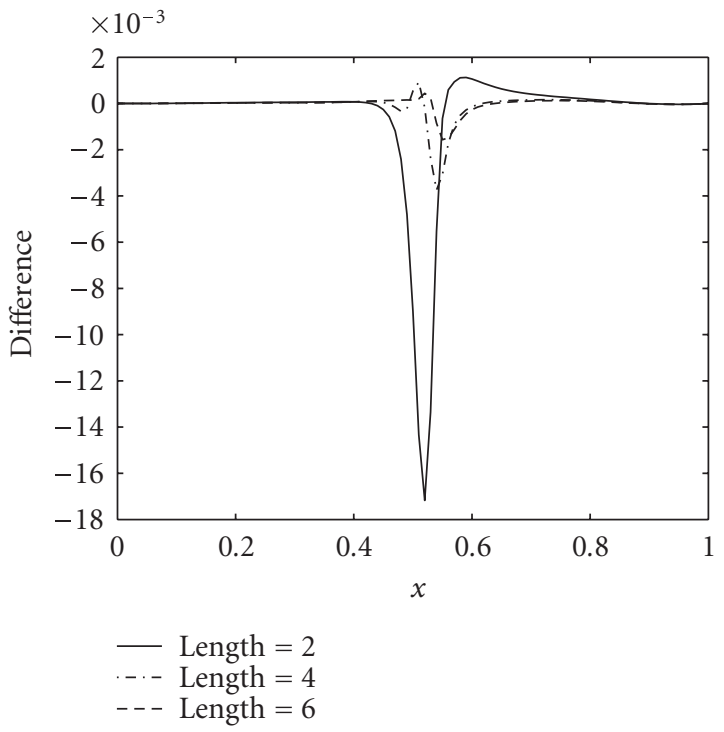

(b)

Figure 4.5. Differences in the first (a) and second (b) moments between the exact solution and approximations from the LW with WCE for several lengths.

WCE is 6 and the Courant number $v=1.0$, the LW with WCE results in $0.06 \%$ of relative error as seen in Figure 4.7. In order to obtain $0.06 \%$ of accuracy, Figure 4.2 shows that the $\mathrm{MC}$ requires more than 50000 realizations. Figure 4.9 represents the CPU time of the MC 
16 Efficient computation of stochastic Burger's equation

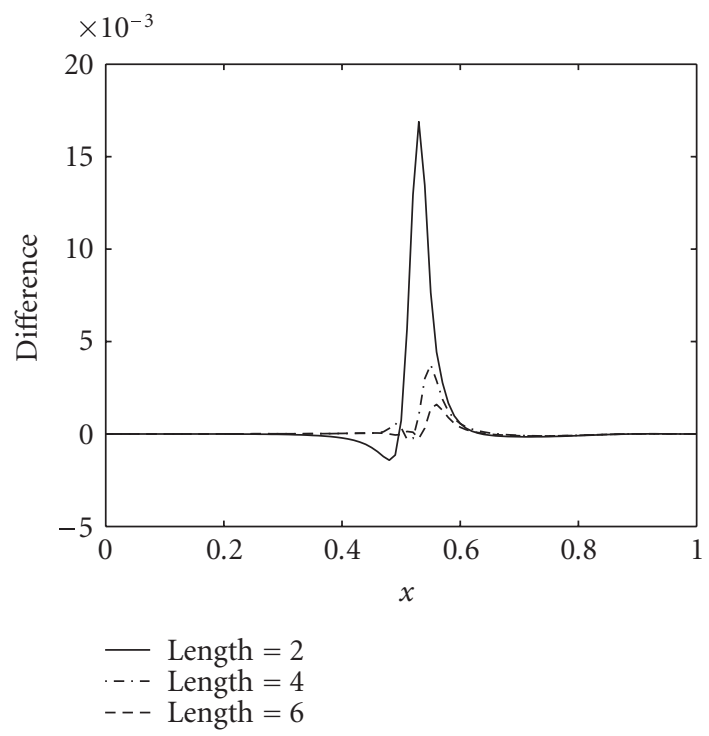

(a)

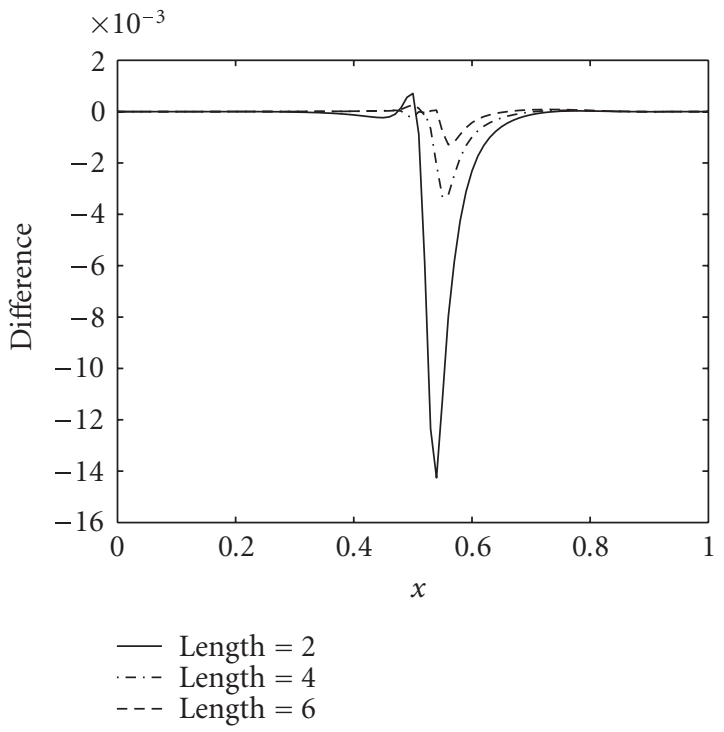

(b)

Figure 4.6. Differences in the third (a) and fourth (b) moments between the exact solution and approximations from the LW with WCE for several lengths.

with respect to the number of realizations and shows that the MC with 50000 samples requires 8.94 seconds of CPU time. 


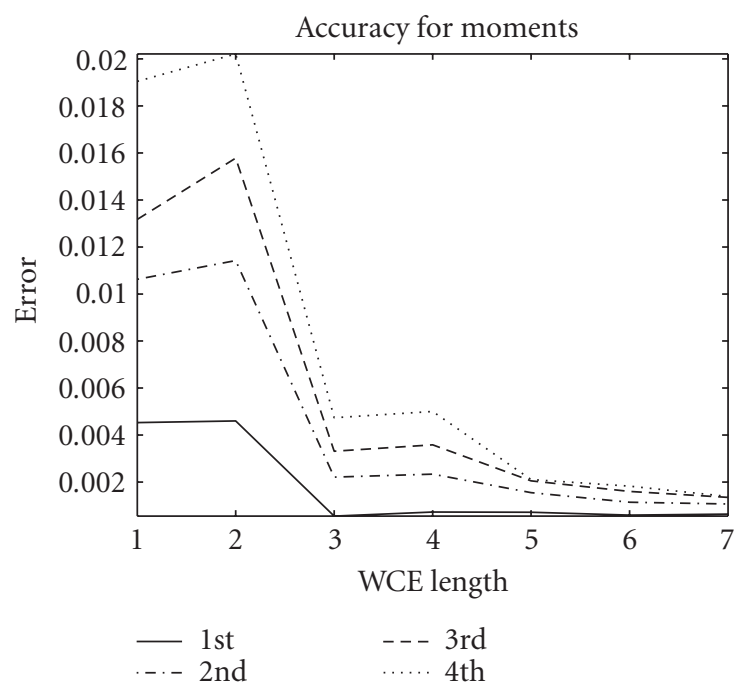

Figure 4.7. Relative errors of the LW with WCE in statistical moments of up to order 4 with respect to the lengths of the WCE.

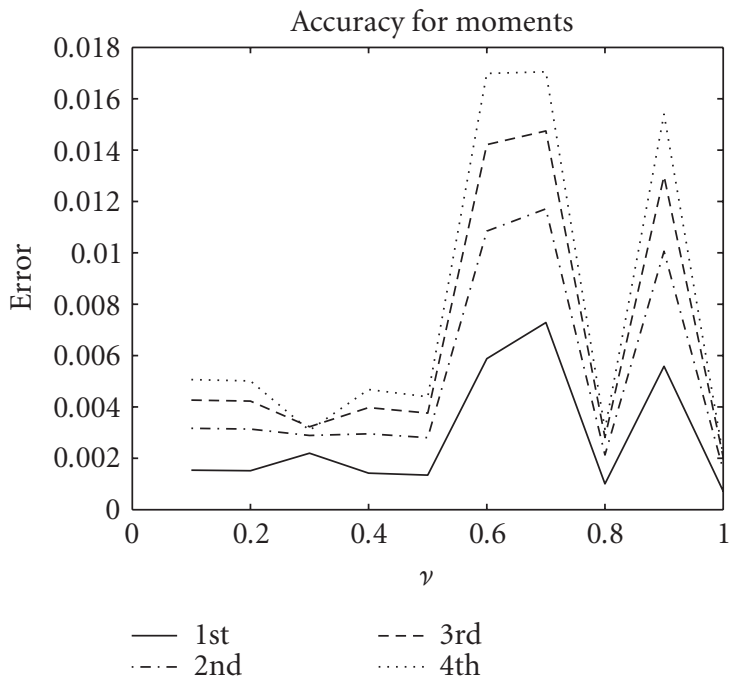

Figure 4.8. Relative errors of the LW with WCE in statistical moments for different Courant numbers.

Figure 4.10 shows the CPU time of the LW with WCE with respect to the lengths of the WCE when the Courant number is $0.6,0.8$, and 1 . It takes 0.67 second with the LW with WCE when the length is 6 and the Courant number is 1.0, which is only $7.49 \%$ of 


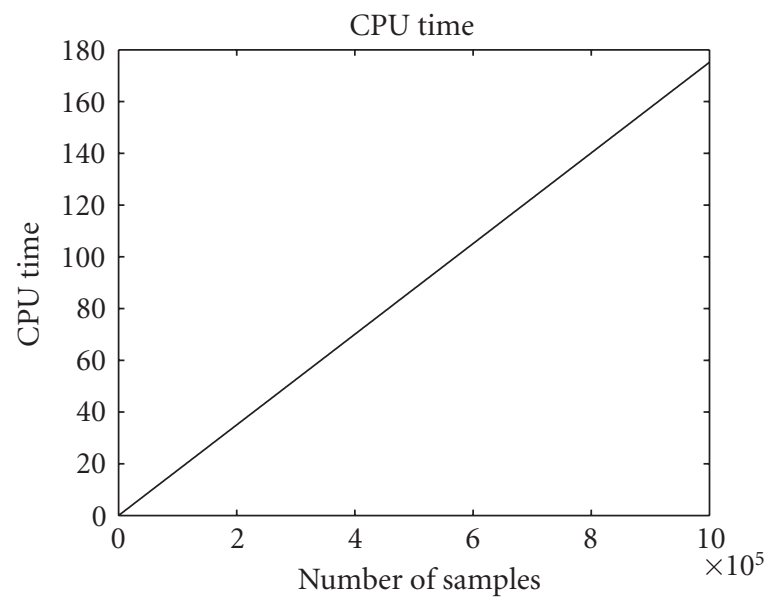

Figure 4.9. CPU time of the $\mathrm{MC}$ with respect to the number of realizations.

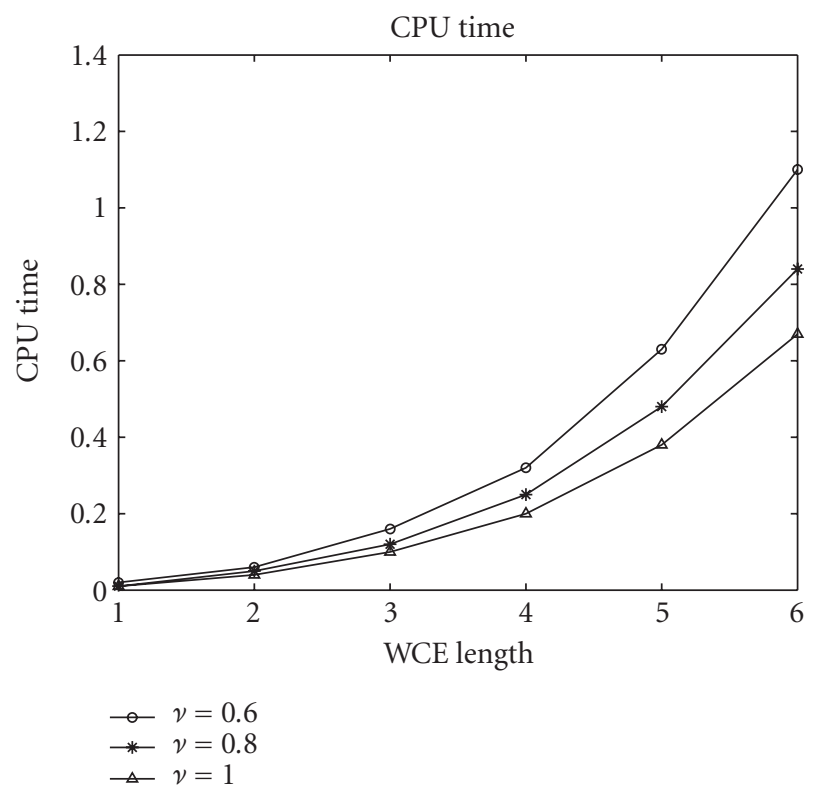

Figure 4.10. CPU time of the LW with WCE as the number of lengths varies when the Courant number is $0.2,0.6$, and 1 .

the computational time, compared to the MC. Figure 4.10 also shows that as the Courant number decreases, $\Delta t$ decreases and more computations are required.

Table 4.2 summarizes the efficiency of the LW with WCE over the MC. Relative error in the table is the error from the LW with WCE when the WCE of a given length is applied. 
Table 4.2. Ratio of CPU times between the MC and the LW with WCE.

\begin{tabular}{lllllll}
\hline WCE length & \multicolumn{1}{c}{1} & \multicolumn{1}{c}{2} & \multicolumn{1}{c}{3} & \multicolumn{1}{c}{4} & \multicolumn{1}{c}{5} & \multicolumn{1}{c}{6} \\
\hline relative error & 0.0045 & 0.0046 & 0.0006 & 0.0007 & 0.0007 & 0.0006 \\
$\mathrm{CPU}_{\mathrm{MC}}$ & 0.35 & 0.35 & 8.94 & 8.58 & 8.58 & 8.94 \\
$\mathrm{CPU}_{\mathrm{LW}}$ & 0.01 & 0.04 & 0.1 & 0.2 & 0.38 & 0.67 \\
$\mathrm{CPU}_{\mathrm{LW}} / \mathrm{CPU}_{\mathrm{MC}}$ & 0.0286 & 0.1143 & 0.0112 & 0.0233 & 0.0443 & 0.0749 \\
\hline
\end{tabular}

$\mathrm{CPU}_{\mathrm{MC}}$ and $\mathrm{CPU}_{\mathrm{LW}}$ in Table 4.2 represent the CPU times taken by the $\mathrm{MC}$ and the $\mathrm{LW}$ with WCE, respectively, to guarantee these relative errors. The table shows that the LW with WCE saves up to $90 \%$ of computational time in the given test problem.

Another advantage of the LW with WCE over the MC is that once the propagator system is solved, since the output is a set of Hermite-Fourier coefficients, a statistical moment of any order can be estimated without any new computation. In case of the MC, however, orders of statistical moments of interest should be decided prior to the computation. For example, if first three moments were obtained during the MC computation, and if the fourth moment is needed after the computation is over, all the MC simulation should be performed again, because the fourth moment in this case cannot be generated from first three moments.

\section{Conclusions}

A version of LW has been derived using the WCE in order to compute efficiently statistical moments of solutions to Burger's equation with random initial conditions. The WCE transforms the LW into an infinite system of deterministic equations with respect to Hermite-Fourier coefficients. One of the important properties of the system is that all the statistical moments of the solution to the Burger's equation can be estimated by simple formulae that involve the solution of the system only. The stability, accuracy, and efficiency of the scheme have been numerically examined and compared to the MC. It has been demonstrated that LW with WCE is equally accurate but substantially faster than $\mathrm{MC}$ at least for certain classes of initial conditions. When the LW with WCE is applied to test problems, the computation time has been reduced up to $90 \%$. In addition, the new approach does not require generation of random numbers and provides a good control over the computational errors. More study on stability and convergence will follow in the future research.

\section{Acknowledgments}

The author would like to thank Boris Rozovskii in University of Southern California for numerous helpful discussions. This research was supported by the Ministry of Information and Communication (MIC), Korea, under the Information Technology Research Center (ITRC) support program, supervised by the Institute of Information Technology Assessment (IITA). 


\section{References}

[1] A. Bensoussan and R. Temam, Équations stochastiques du type Navier-Stokes, Journal of Functional Analysis 13 (1973), no. 2, 195-222.

[2] Z. Brzeźniak, M. Capiński, and F. Flandoli, Stochastic partial differential equations and turbulence, Mathematical Models \& Methods in Applied Sciences 1 (1991), no. 1, 41-59.

[3] R. H. Cameron and W. T. Martin, The orthogonal development of non-linear functionals in series of Fourier-Hermite functionals, Annals of Mathematics. Second Series 48 (1947), 385-392.

[4] M. Capiński and T. Zastawniak, Mathematics for Finance, Springer Undergraduate Mathematics Series, Springer, London, 2003.

[5] A. J. Chorin, Hermite expansions in Monte-Carlo computation, Journal of Computational Physics 8 (1971), no. 3, 472-482.

[6]__ Gaussian fields and random flow, Journal of Fluid Mechanics 63 (1974), no. 1, 21-32.

[7] S. C. Crow and G. H. Canavan, Relationship between a Wiener-Hermite expansion and an energy cascade, Journal of Fluid Mechanics 41 (1970), 387-403.

[8] A. De Bouard, A. Debussche, and Y. Tsutsumi, Periodic solutions of the Korteweg-de Vries equation driven by white noise, SIAM Journal on Mathematical Analysis 36 (2004), no. 3, 815-855.

[9] W. E and Ya. G. Sină̌, New results in mathematical and statistical hydrodynamics, Russian Mathematical Surveys 55 (2000), no. 4, 635-666.

[10] R. J. Elliott and P. E. Kopp, Mathematics of Financial Markets, Springer Finance, Springer, New York, 2005.

[11] J. E. Gentle, Random Number Generation and Monte Carlo Methods, Statistics and Computing, Springer, New York, 2003.

[12] R. G. Ghanem and P. D. Spanos, Stochastic Finite Elements: A Spectral Approach, Springer, New York, 1991.

[13] J. Glimm, S. Hou, H. Kim, Y.-h. Lee, D. H. Sharp, K. Ye, and Q. Zou, Risk management for petroleum reservoir production: a simulation-based study of prediction, Computational Geosciences 5 (2001), no. 3, 173-197.

[14] J. Glimm, S. Hou, H. Kim, D. Sharp, and K. Ye, A probability model for errors in the numerical solutions of a partial differential equation, Computational Fluid Dynamics Journal 9 (2000), 485493.

[15] J. Glimm and A. Jaffe, Quantum Physics. A Functional Integral Point of View, 2nd ed., Springer, New York, 1987.

[16] T. Y. Hou, H. Kim, B. Rozovskii, and H. Zhou, Wiener chaos expansions and numerical solutions of randomly forced equations of fluid mechanics, HERMIS 4 (2003), 1-14.

[17] M. Jardak, C.-H. Su, and G. E. Karniadakis, Spectral polynomial chaos solutions of the stochastic advection equation, Journal of Scientific Computing 17 (2002), no. 1-4, 319-338.

[18] R. H. Kraichnan, Small-scale structure of a scalar field convected by turbulence, Physics of Fluids 11 (1968), no. 5, 945-953.

[19] E. Kreyszig, Introductory Functional Analysis with Applications, Wiley Classics Library, John Wiley \& Sons, New York, 1989.

[20] L. D. Landau and E. M. Lifshitz, Fluid Mechanics, Course of Theoretical Physics, vol. 6, Pergamon Press, London, 1959.

[21] R. J. LeVeque, Finite Volume Methods for Hyperbolic Problems, Cambridge Texts in Applied Mathematics, Cambridge University Press, Cambridge, 2002.

[22] J. S. Liu, Monte Carlo Strategies in Scientific Computing, Springer Series in Statistics, Springer, New York, 2001.

[23] P.-A. Meyer, Quantum Probability for Probabilists, Lecture Notes in Mathematics, vol. 1538, Springer, Berlin, 1993.

[24] R. Mikulevicius and B. L. Rozovskii, Linear parabolic stochastic PDEs and Wiener chaos, SIAM Journal on Mathematical Analysis 29 (1998), no. 2, 452-480. 
[25] _ Martingale problems for stochastic PDE's, Stochastic Partial Differential Equations: Six Perspectives, Math. Surveys Monogr., vol. 64, American Mathematical Society, Rhode Island, 1999, pp. 243-325.

[26] Stochastic Navier-Stokes equations for turbulent flows, SIAM Journal on Mathematical Analysis 35 (2004), no. 5, 1250-1310.

[27] G. N. Milstein and M. V. Tretyakov, Evaluation of conditional Wiener integrals by numerical integration of stochastic differential equations, Journal of Computational Physics 197 (2004), no. 1, 275-298.

[28] K. W. Morton and D. F. Mayers, Numerical Solution of Partial Differential Equations, Cambridge University Press, Cambridge, 1994.

[29] G. Sansone, Orthogonal Functions, Dover, New York, 1991.

[30] M. Scalerandi, A. Romano, and C. A. Condat, Korteweg-de Vries solitons under additive stochastic perturbations, Physical Review E 58 (1998), no. 4, 4166-4173.

[31] R. Spigler, Monte Carlo-type simulation for solving stochastic ordinary differential equations, Mathematics and Computers in Simulation 29 (1987), no. 3-4, 243-251.

[32] J. C. Tannehill, D. A. Anderson, and R. H. Pletcher, Computational Fluid Mechanics and Heat Transfer, Taylor \& Francis, Washington, DC, 1997.

[33] D. Zhang and Z. Lu, An efficient high-order perturbation approach for flow in random porous media via Karhunun-Loeve and polynomial expansion, Journal of Computational Physics 194 (2004), no. 2, 773-794.

Hongjoong Kim: Department of Mathematics, Korea University, 1 Anamdong, Sungbuk-ku, Seoul 136-701, South Korea

E-mail address: hongjoong@korea.ac.kr 


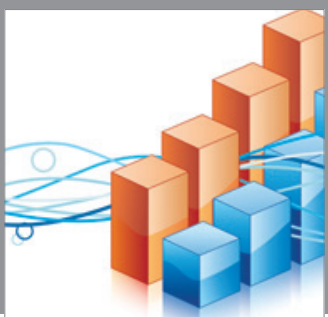

Advances in

Operations Research

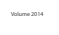

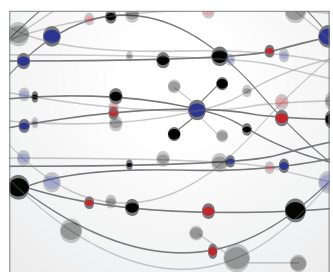

\section{The Scientific} World Journal
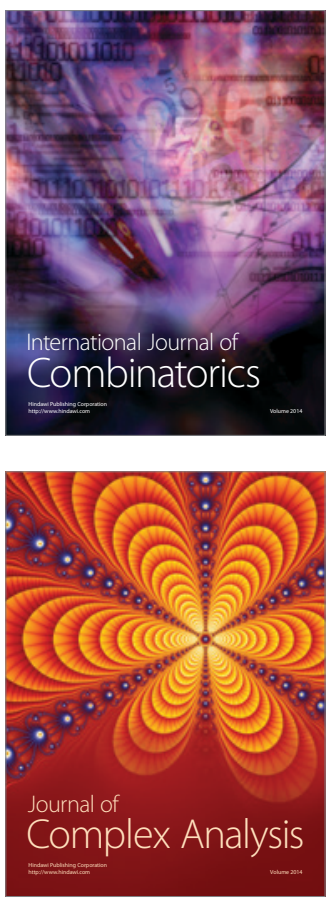

International Journal of

Mathematics and

Mathematical

Sciences
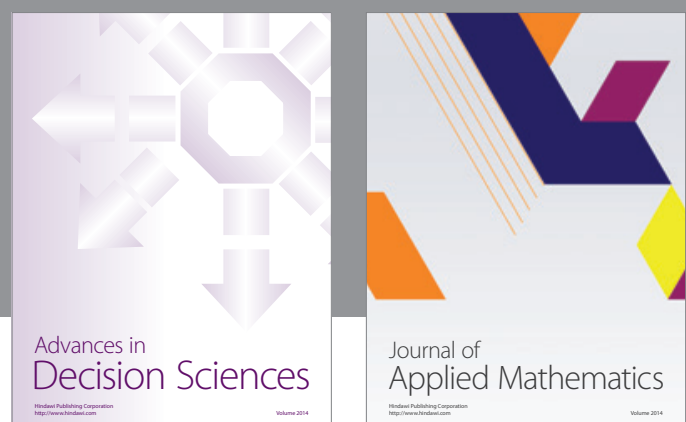

Journal of

Applied Mathematics
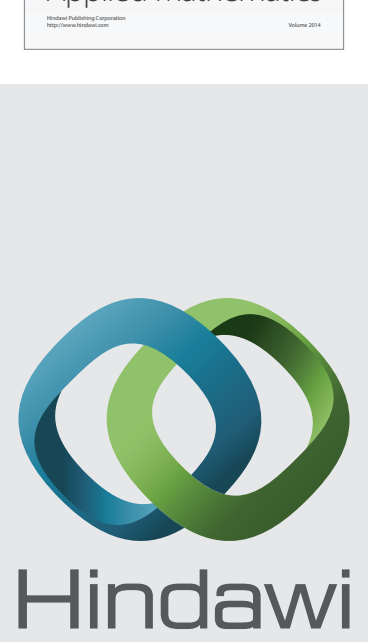

Submit your manuscripts at http://www.hindawi.com
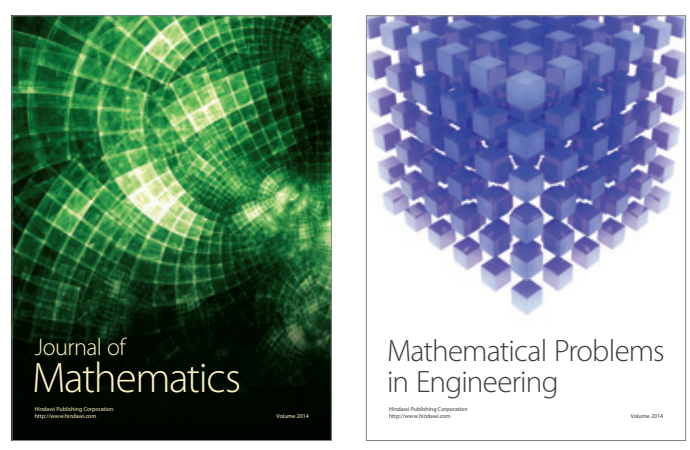

Mathematical Problems in Engineering
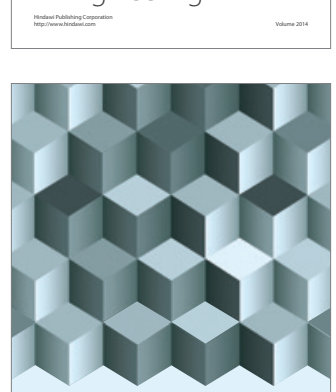

Journal of

Function Spaces
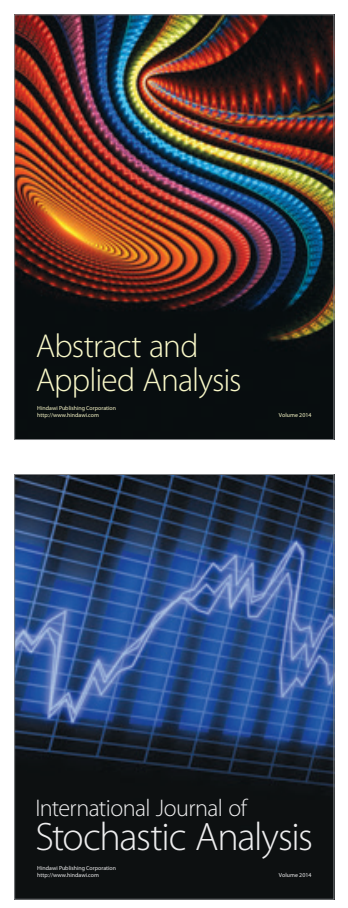

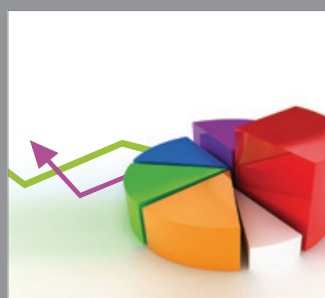

ournal of

Probability and Statistics

Promensencen
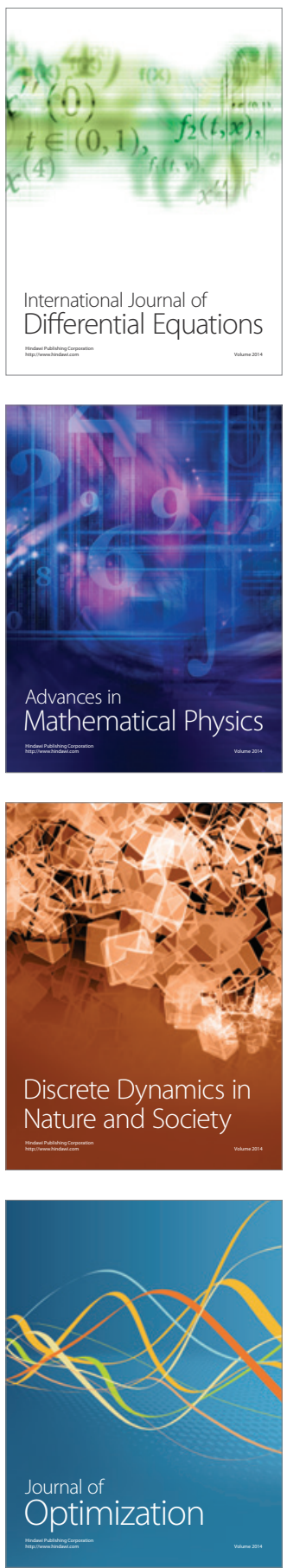